\title{
Caracterización de adultos sin riesgo, con riesgo y con diabetes en el noreste de México
}

\author{
Ramírez-Girón, Nataliai*; Gallegos-Cabriales, Esther²
}

\section{RESUMEN}

Objetivo: Caracterizar un grupo de adultos sin riesgo, con riesgo de diabetes tipo 2 y con diabetes tipo 2 en el noreste de México. Método: Diseño descriptivo, correlacional y transversal; se incluyeron 250 personas aparentemente sanas, de ambos sexos, entre 18 a 65 años de edad, reclutadas en un centro comercial de Monterrey, México. Se aplicaron instrumentos de lápiz y papel, mediciones antropométricas y muestra de $3 \mathrm{ml}$ de sangre para procesamiento de hemoglobina glucosilada.

Resultados: predominó el sexo femenino (72.8\%), la media de edad fue 43.9 años ( $D E=12.7$ ), promedio de escolaridad 11.5 años $(D E=4.3)$, 80\% tienen antecedentes familiares de diabetes; $13.1 \%$ de los participantes tuvieron cifras indicativas de diabetes y $26.9 \%$ riesgo de desarrollarla. Los resultados de porcentaje de grasa y circunferencia de cintura estuvieron por encima de los puntos de corte recomendados. Se percibió un alto riesgo de diabetes, alto apoyo social, ingreso mensual suficiente y características comunitarias favorecedoras para la salud; por el contrario la ansiedad, el estilo de vida saludable, los síntomas depresivos y el estrés laboral tuvieron bajos porcentajes. El 86.8\% asisten al servicio médico solo cuanto están enfermos y a más del $50 \%$ se le recomienda sobre hacer ejercicio y mantener una alimentación saludable; a más de la mitad se les informó tener riesgo para diabetes. Conclusión: Es importante la utilización de la perspectiva ecológica que apertura la comprensión de los procesos y factores de diferentes niveles de organización social involucrados en el riesgo de desarroIlar DMT2.

Palabras clave: Diabetes Mellitus; Salud Pública; Ecología Social; Factores de Riesgo (DeCS, BIREME).

${ }^{1}$ Maestría en Enfermería, Facultad de Enfermería, Universidad Autónoma de Nuevo León, Monterrey.ORCID-ID: 0000-0002-8312-6287. E-mail:
natalia.ramirez@hotmail.com
${ }^{2}$ Doctorado en Ciencias de Enfermería, Facultad de Enfermería, Universidad Autónoma de Nuevo León, Monterrey. ORCID-ID: 0000-0003-3619-2596

Recibido: 05/06/2019

Aceptado: 30/06/2019

${ }^{\star}$ Autor para correspondencia

\section{Cómo citar este artículo}

Ramírez-Girón N, Gallegos-Cabriales E. Caracterización de adultos sin riesgo, con riesgo y con diabetes en el noreste de México. SANUS. 2019;(10): 33-51. [Acceso_- $\frac{\text { ] }}{\text { ] }}$; Disponible en: mes día año URL 


\title{
Caracterização de adultos sem risco e com risco de desenvolver diabetes tipo 2 e adultos com diabetes tipo 2 no norte do México
}

\section{ABSTRATO}

\begin{abstract}
Introdução: Caracterizar um grupo de adultos sem risco e com risco de desenvolver diabetes tipo 2 e com diabetes tipo 2 no norte do México Metodologia: Projeto descritivo, correlational e transversal; 250 pessoas aparentemente saudáveis, do sexo masculino e femenino, entre 18 e 65 anos de idades, foram incluídas, que foram recrutados em um shopping center de Monterrey, México. Os instrumentos utilizados foram papel e lápis, medidas antropométricas e uma amostra de $3 \mathrm{ml}$ de sangue para processar a hemoglobina glicada. Resultados: as fêmeas predominaram (72,8\%), a idade média foi de 43,9 anos $(\mathrm{DE}=12.7)$ e uma media de 11,5 anos de escolaridade ( $\mathrm{DE}=4.3), 80 \%$ tem histórico familiar de diabetes; $13,1 \%$ dos participantes tinham valores indicativos de diabetes, e 26,9\% tinham o risco de desenvolvê-lo. Os resultados quanto ao percentual de gordura e circunferência da cintura ficaram acima dos pontos de corte recomendados. Um alto risco de diabetes, alto apoio social, renda mensal suficiente e características da comunidade favoráveis à saúde foram notados; pelo contrário, ansiedade, estilo de vida saudável, sintomas de depressão e estresse relacionado ao trabalho tiveram baixas porcentagens. 86,8\% de pessoas só vêem o médico quando estão doentes, e mais de 50\% das pessoas foram recomendadas para se exercitar e manter uma dieta balanceada; mais da metade das pessoas foram informadas de estar em risco de diabetes. Conclusões: importante usar a perspective ecológica que abre a compreensão de processos e fatores de diferentes níveis de organização social envolvidos no risco de desenvolver DM2.
\end{abstract}

\section{Palavras chave: Diabetes mellitus; saúde pública; ecologia social; fatores de risco (DeCS;BIREME)}

\section{INTRODUCCIÓN}

La Diabetes Mellitus Tipo 2 (DM2) es un problema de salud pública mundial(1); en México es la segunda causa de muerte, con un incremento del $4 \%$ en la prevalencia durante los últimos cuatro años lo que ha llevado al país a ocupar el quinto lugar a nivel mundial con más adultos diagnosticados de $\mathrm{DM}^{(2-4)}$. Pese a la existencia de múltiples normas e intervenciones de prevención y detección temprana del riesgo de DM2 a nivel nacional(5,6) la implementación de éstas y el cumplimiento de los indicadores de prevención están aún lejos de alcanzarse; algunos autores afirman que la DM2 ha tenido un patrón de crecimiento positivo de $2.7 \%$ cada año(7).

La evidencia sugiere que este patrón de crecimiento se debe a la naturaleza metabólica compleja, ya que se asocia con factores no modificables (edad, raza, herencia genética), y factores modificables relacionados con estilos de vida poco saludables (sedentarismo, sobrepeso y obesidad [SP/OB], dieta poco saludable y consumo de alcohol) ${ }^{(2,8)}$. La literatura reporta diversas intervenciones exitosas las cuales han manipulado los factores individuales, como cambios en el patrón de alimentación y el fomento de la actividad física. Sin embargo, aunque se han reportado resultados significativos, éstos no han impactado en la disminución de la incidencia y prevalencia a nivel nacional(7).
Diferentes posturas teóricas ${ }^{(11,12)}$ entre ellas el Modelo Ecológico para la Promoción de la Salud (MEPS)(13) sustenta que el desarrollo del riesgo y la progresión hacia la enfermedad es producto de la presencia de factores en diferentes niveles de la estructura social, como lo son, nivel interpersonal (relaciones interpersonales), nivel institucional (condiciones de trabajo), nivel comunitario (circunstancias específicas de vida) y nivel de política pública (acceso y uso de acciones a nivel público). En este sentido, es indispensable la comprensión de los diferentes factores involucrados en la progresión del riesgo de DM2 hasta la aparición plena de la enfermedad a partir de la perspectiva ecológica, incluyendo factores que no han sido estudiados a profundidad, dado que pueden influir en el individuo y hacerlo vulnerable a desarrollar la enfermedad.

La revisión de literatura pone en evidencia factores individuales como falta de ejercicio, SP/OB, HTA, antecentes familiares de DM22, bajo nivel educativo y económico(14), ansiedad, depresión ${ }^{(15)}$, consumo de sustancias psicoactivas $s^{(16)}$, baja percepción de riesgo ${ }^{(17) ;}$ así como factores en diferentes niveles de estructura social como el escaso apoyo social(18), extensas jornadas de trabajo, estrés laboral ${ }^{(19,20)}$, inseguridad alimentaria, percepción de problemas comunitarios ${ }^{(21,22)}$, desarrollo urbano y urbanización ${ }^{(23-25)}$ el bajo nivel educativo en la comunidad(26), bajo acceso a la atención en el sistema sanitario ${ }^{(27,28)}$ y la economía local y nacional ${ }^{(29,30)}$. 
Hasta el momento, no se ha encontrado evidencia sobre factores individuales y de la estructura social, en el marco de una perspectiva teórica como el MEPS, que estén presentes e influyan sobre el riesgo de DM2 y la progresión a la enfermedad en el noreste de México; además, dada la magnitud que este problema representa es esencial contribuir con la mejor comprensión de los factores ecológicos que influyen en el desarrollo de la DM2, permitir el re-diseño y la implementación de las intervenciones para prevenir la enfermedad con mayor probabilidad de reducir la incidencia, la prevalencia y los costos adicionales asociados al tratamiento a nivel local. De acuerdo con lo anterior, el objetivo del estudio fue caracterizar un grupo de adultos sin riesgo, con riesgo de diabetes tipo 2 y con diabetes tipo 2 en el noreste de México.

\section{METODOLOGÍA}

Diseño descriptivo transversal, la población fueron personas entre 18 a 65 años, de ambos sexos provenientes del área metropolitana de Monterrey, México. El tamaño de muestra se calculó mediante nQuery Advisor con una significancia estadística $(\alpha)<.05$, magnitud del efecto $(\gamma)$ mediano y poder (1- $\beta$ ) de .90, para una muestra de 250 participantes, el muestreo fue no probabilístico. Se incluyeron personas de ambos sexos, entre 18 a 65 años; se excluyeron personas con Diabetes Mellitus tipo 1, Diabetes de la Edad Madura en el Joven (MODY) y mujeres en gestación.

Los participantes fueron reclutados en un centro comercial del área metropolitana de Monterrey mediante invitación personal y anuncios escritos. A las personas que estuvieron de acuerdo en participar, se les brindó una explicación detallada sobre el estudio. Dada la aprobación por el participante, se verificó el cumplimiento de los criterios de inclusión y se realizó el proceso de consentimiento informado. La recolección de datos fue realizada en tres fases: 1. El llenado de los instrumentos, 2. Las mediciones antropométricas y 3. La extracción de la muestra sanguínea para procesamiento de la Hemoglobina Glucosilada (HbA1c). Este proceso fue realizado por una estudiante de doctorado en ciencias de enfermería y una auxiliar de investigación, previamente capacitadas y entrenadas para el llenado de los instrumentos, toma de mediciones antropométricas y la extracción de $3 \mathrm{ml}$ de sangre venosa. Esta última fue procesada por el laboratorio de análisis clínico "Q.F.B. Iris Guajardo Guajardo" de la Facultad de Ciencias Químicas de la Universidad Autónoma de Nuevo León (Certificación ISO - 9001:2008), mediante Cromatografía Líquida de Alta Resolución (HPLC), de acuerdo con lineamientos estandarizados internacionales2. La HbA1c se clasificó de acuerdo a la Asociación Americana de Diabetes2, cifras $\leq$ $5.6 \%$ se consideraron normales, de $5.7 \%$ a $6.4 \%$ HbA1c de riesgo para DM2 y $\geq 6.5 \% \mathrm{HbA} 1 \mathrm{c}$ indicativa de DM2.

El estudio se realizó bajo lineamientos éticos establecidos en la Ley General de Salud en Materia de Investigación para la Salud Mexicana ${ }^{(31)}$, así como la aprobación por parte de un Comité de Bioética, Investigación y Bioseguridad, con el número de registro D-1325. En todos los casos se obtuvo el consentimiento informado de forma previa al llenado de los instrumentos. Los datos fueron colectados en el segundo semestre del año 2018.

Las variables para medir los niveles teóricos correspondientes al individuo y la estructura social según el MEPS, se describen en la tabla 1, todos los instrumentos han sido validados en población mexicana. Para el análisis estadístico, las variables continuas se presentan con medias y desviación estándar (s) y las categóricas con frecuencias y porcentajes.

\section{RESULTADOS}

La HbA1c se determinó en 245 participantes, dado que en cinco participantes no fue posible extraer la muestra sanguínea. El 60\% tiene cifras de HbA1c dentro de los límites normales, 26.9\% presentan cifras de riesgo para DM2 y $13.1 \%$ cifras indicativas de DM2.

Predominó el sexo femenino con $72.8 \%$; la media de edad fue 43.9 años $(s=12.7)$; la escolaridad promedio fue 11.5 años $(s=4.3)$. El 80.8\% refirieron tener antecedentes familiares de DM2. El 32.8\% y $17.2 \%$ de los participantes consumen bebidas alcohólicas y cigarrillos respectivamente, siendo mayor el consumo en hombres que en mujeres.

La tabla 2 muestra que, quienes tienen riesgo de DM2 o ya tienen DM2 cuentan con la mayor proporción de familiares que padecen la enfermedad; predomina la obesidad y la alta percepción de factores de riesgo para DM2; la mayor parte de la muestra no practíca estilos de vida saludables; la ansiedad y los sintomas depresivos se presentaron en bajo porcentajes.

De acuerdo con la tabla 3, la mayoría de participantes perciben un alto apoyo social, un ingreso mensual suficiente para cubrir sus necesidades básicas, bajo estrés laboral y características comunitarias favorecedoras para la salud. Casi la totalidad del grupo estudiado, asisten al servicio de salud sólo cuando se sienten enfermos; a más de la mitad de la muestra se les ha recomendado hacer ejercicio y mantener alimentación saludable.

\section{DISCUSIÓN}

La DM2 requiere una comprensión más amplia de todos los factores que intervienen en el desarrollo del riesgo y la progresión hacia la enfermedad desde perspectivas teóricas que superen el enfoque circunscrito al individuo. Por esto, es esencial la inclusión del MEPS que establece la importancia de variables fuera del individuo y en diferentes niveles de organización social que pueden determinar las conductas promotoras de salud. Este abordaje teórico es de importancia para la disciplina de enfermería por extender el alcance de variables interactuantes que influyen en el riesgo y el desarrollo pleno de la DM2; padecimiento que impacta significativamente a la población mexicana ${ }^{(23)}$.

La muestra reclutada se integró en su mayoría por mujeres, lo que coincide con otros autores ${ }^{(14,16,22),}$ 
Tabla 1. Definición y operacionalización de las variables

\begin{tabular}{|c|c|}
\hline \multicolumn{2}{|r|}{ Niveles de influencia } \\
\hline \multicolumn{2}{|r|}{ Nivel intrapersonal } \\
\hline Sexo & Femenino, Masculino. \\
\hline Edad & Años de vida cumplidos \\
\hline Nivel de educación & Años de formación académica realizados. \\
\hline $\begin{array}{l}\text { Antecedentes fami- } \\
\text { liares de DMT2 }\end{array}$ & Diagnóstico de DMT2 en uno o más miembros de la familia \\
\hline Consumo de alcohol & Ingestión voluntaria de alcohol. Si/No. \\
\hline $\begin{array}{l}\text { Consumo de Ciga- } \\
\text { rrillo }\end{array}$ & Ingestión voluntaria de cigarrillo. Si/No. \\
\hline $\begin{array}{l}\text { Circunferencia de } \\
\text { Cintura (CC) }\end{array}$ & CC aumentada en mujeres $>80$ cm y en hombres $>94$ cm. Se midió con cinta métrica flexible SECA 203. \\
\hline Grasa corporal & Aumentada > 25\% en hombres y > 35\% en mujeres. Se midió con la báscula InBody 230. \\
\hline Estilo de vida & $\begin{array}{l}\text { Acciones individuales dirigidas a promover un estilo de vida saludable. Se evaluó con el "Cuestionario de Perfil de Estilo de } \\
\text { Vida II" (PEPS-II)" (32). }\end{array}$ \\
\hline $\begin{array}{l}\text { Percepción de riesgo } \\
\text { de DMT2 }\end{array}$ & $\begin{array}{l}\text { Identificación individual de características y comportamientos que pueden aumentar el riesgo de DMT2. Se evaluó con el } \\
\text { instrumento "Percepción de los Factores de Riesgo de Diabetes Tipo 2"(33). }\end{array}$ \\
\hline Ansiedad & $\begin{array}{l}\text { Respuesta emocional transitoria o permanente con síntomas somáticos de tensión frente a estímulos percibidos como } \\
\text { amenazantes. Se evaluó con el "Cuestionario de Ansiedad Estado-Rasgo" (STAI) }{ }^{(34)} \text {. }\end{array}$ \\
\hline Síntomas depresivos & $\begin{array}{l}\text { Sentimientos de tristeza, desesperación y disminución de la capacidad para disfrutar de actividades preferidas. Se evaluó } \\
\text { con el "Inventario de Depresion de Beck II" (BDI-II) }{ }^{(35)} \text {. }\end{array}$ \\
\hline \multicolumn{2}{|r|}{ Interpersonal Level } \\
\hline Social support & $\begin{array}{l}\text { Percepción de disponibilidad de una persona para proveer ayuda o apoyo emocional, físico y económico. Se evaluó con la } \\
\text { "Escala Multidimensional de Apoyo Social Percibido" (MSPSS) }{ }^{\left({ }^{(6)} \text {. }\right.}\end{array}$ \\
\hline \multicolumn{2}{|r|}{ Institutional Level } \\
\hline Monthly income & Salario económico mensual para suplir las necesidades básicas. Suficiente/ No suficiente. \\
\hline Work-related stress & $\begin{array}{l}\text { Estado mental de presión y preocupación relacionada con alguna actividad laboral. Se evaluó con el "Cuestionario Desba- } \\
\text { lance Esfuerzo / Recompensa"(37). }\end{array}$ \\
\hline \multicolumn{2}{|r|}{ Community Level } \\
\hline $\begin{array}{l}\text { Características de la } \\
\text { comunidad }\end{array}$ & $\begin{array}{l}\text { Disponibilidad comunitaria de alimentación saludable, zonas verdes, espacios de deporte, y seguridad los cuales pueden } \\
\text { beneficiar la salud. Se evaluó con el instrumento "Características de la Comunidad"(38). }\end{array}$ \\
\hline \multicolumn{2}{|r|}{ Public Policy Level } \\
\hline Política pública & $\begin{array}{l}\text { Acceso y uso de las acciones en salud relativas al control de peso, actividad física, plan de alimentación saludable, educa- } \\
\text { ción sobre factores de riesgo, consultas multidisciplinarias y pruebas de laboratorio para prevenir el riesgo de DMT2 }{ }^{(39)} \text {. }\end{array}$ \\
\hline
\end{tabular}

\section{Source: Self-source.}

confirmando que, es el sexo femenino quien muestra mayor interés en su salud. Llama la atención, el porcentaje muy por encima de lo reportado a nivel nacional de participantes con antecedentes familiares de DM2 ${ }^{(14)}$. La evidencia demuestra que estas personas tienen hasta un 30\% de probabilidades de padecer DM2 en edades más tempranas, en comparación con quienes no tienen estos antecedentes; esto relacionado probablemente con genes ligados a la susceptibilidad para $\mathrm{DM} 2^{(40)}$. En esta muestra, esta condición de riesgo de DM2 se explica además por el estado de SP/OB (más de cinco puntos porcentuales que la prevalencia nacional) del grupo participante, considerado factor de alto riesgo para DM2 ${ }^{(14)}$.

Un factor positivo en este grupo fue, el promedio de escolaridad por encima de la media nacional(3). Se sabe que el nivel educativo se asocia con el riesgo de DM2; diversos estudios han mostrado que a menor nivel educativo, el riesgo e incidencia de DM2 se incrementa. Lo opuesto también se ha confirmado; un nivel alto de escolaridad favorece la adopción de comportamientos saludables que disminuyen la probabilidad de desarrollar la enfermedad(16).

La mayoría de los participantes manifestó no consumir alcohol, ni fumar cigarrillos; situación que no coincide con la literatura que sustenta fuerte asociación del consumo de este tipo de drogas con el riesgo y la incidencia de DM2 $2^{(16,18)}$. Este comportamiento en la muestra puede deberse al alto nivel educativo considerado como factor protector de la salud, ya que permite la toma de decisiones asertivas evitando circunstancias y situaciones de riesgo para la salud.

Las cifras de $\mathrm{HbA1c}$ en personas adultas sanas sugieren que la población del noreste del país es proclive a desarrollar DM2(14). Esta situación se explica por la susceptibilidad heredada; las conductas y estilos de vida 
Tabla 2. Frecuencias y porcentajes de variables intrapersonales según cifras de HbA1c

\begin{tabular}{|c|c|c|c|c|c|c|c|c|}
\hline \multirow{3}{*}{ Variables } & \multicolumn{2}{|c|}{ Total } & \multicolumn{2}{|c|}{ Sin riesgo de DMT2 } & \multicolumn{2}{|c|}{$\begin{array}{c}\text { Con riesgo de } \\
\text { DMT2 }\end{array}$} & \multicolumn{2}{|c|}{ Con DMT2 } \\
\hline & \multirow[t]{2}{*}{$f$} & \multirow[t]{2}{*}{$\%$} & $f$ & $\%$ & $f$ & $\%$ & $f$ & $\%$ \\
\hline & & & 147 & 60 & 66 & 26.9 & 32 & 13.1 \\
\hline Antecedentes familiares & & & & & & & & \\
\hline $\mathrm{Si}$ & 197 & 80.4 & 110 & 74.80 & 59 & 89.4 & 28 & 87.5 \\
\hline No & 35 & 14.3 & 26 & 17.70 & 5 & 7.6 & 4 & 12.5 \\
\hline No se sabe & 13 & 5.3 & 11 & 7.50 & 2 & 3.0 & 0 & 0 \\
\hline Total & 245 & 100 & 147 & 100.00 & 66 & 100 & 32 & 100 \\
\hline $\begin{array}{l}\text { Circunferencia de Cintura } \\
\text { Hombres }\end{array}$ & & & & & & & & \\
\hline Normal & 21 & 31.3 & 15 & 33.3 & 3 & 20 & 3 & 42.9 \\
\hline Aumentada & 46 & 68.7 & 30 & 66.7 & 12 & 80 & 4 & 57.1 \\
\hline Total & 67 & 100 & 45 & 100 & 15 & 100 & 7 & 100 \\
\hline Mujeres & & & & & & & & \\
\hline Normal & 23 & 13 & 12 & 11.8 & 6 & 11.8 & 5 & 20 \\
\hline Aumentada & 155 & 87 & 90 & 88.2 & 45 & 88.2 & 20 & 80 \\
\hline Total & 178 & 100 & 102 & 100 & 51 & 100 & 25 & 100 \\
\hline $\begin{array}{l}\text { Porcentaje de grasa corporal } \\
\text { Hombres }\end{array}$ & & & & & & & & \\
\hline Normal & 19 & 28.4 & 15 & 33.3 & 2 & 13.3 & 2 & 28.6 \\
\hline Aumentada & 48 & 71.6 & 30 & 66.7 & 13 & 86.7 & 5 & 71.4 \\
\hline Total & 67 & 100 & 45 & 100 & 15 & 100 & 7 & 100 \\
\hline Mujeres & & & & & & & & \\
\hline Normal & 33 & 18.5 & 21 & 20.6 & 8 & 15.7 & 4 & 16 \\
\hline Aumentada & 145 & 81.5 & 81 & 79.4 & 43 & 84.3 & 21 & 84 \\
\hline Total & 178 & 100 & 102 & 100 & 51 & 100 & 25 & 100 \\
\hline Estilo de Vida & & & & & & & & \\
\hline Desarrollados & 88 & 35.9 & 51 & 34.7 & 24 & 36.4 & 13 & 40.6 \\
\hline No desarrollados & 157 & 64.1 & 96 & 65.3 & 42 & 63.6 & 19 & 59.4 \\
\hline Total & 245 & 100 & 147 & 100 & 66 & 100 & 32 & 100 \\
\hline Percepción de Riesgo de DMT2 & & & & & & & & \\
\hline Alta & 163 & 66.5 & 88 & 59.8 & 53 & 80.3 & 22 & 68.7 \\
\hline Baja & 82 & 33.5 & 59 & 40.2 & 13 & 19.7 & 10 & 31.3 \\
\hline Total & 245 & 100 & 147 & 100 & 66 & 100 & 32 & 100 \\
\hline Ansiedad & & & & & & & & \\
\hline Alta & 25 & 10.2 & 19 & 12.9 & 4 & 6.1 & 2 & 6.3 \\
\hline Baja & 220 & 89.8 & 128 & 87.1 & 62 & 93.9 & 30 & 93.7 \\
\hline Total & 245 & 100 & 147 & 100 & 66 & 100 & 32 & 100 \\
\hline Síntomas Depresivos & & & & & & & & \\
\hline Mínimos & 157 & 64.1 & 93 & 63.3 & 45 & 68.2 & 19 & 59.4 \\
\hline Ligeros & 34 & 13.9 & 18 & 12.2 & 11 & 16.7 & 5 & 15.6 \\
\hline Moderados & 33 & 13.5 & 21 & 14.3 & 7 & 10.6 & 5 & 15.6 \\
\hline Severos & 21 & 8.5 & 15 & 10.2 & 3 & 4.5 & 3 & 9.4 \\
\hline Total & 245 & 100 & 147 & 100 & 66 & 100 & 32 & 100 \\
\hline
\end{tabular}

Fuente: Elaboración propia

$\boldsymbol{n}=245$ 
Tabla 3. Frecuencias y porcentajes de variables interpersonales, institucionales, comunitarias y de política pública según cifras de HbA1c

\begin{tabular}{|c|c|c|c|c|c|c|c|c|c|}
\hline \multirow{3}{*}{$\begin{array}{c}\text { Nivel de } \\
\text { influencia }\end{array}$} & \multirow[t]{3}{*}{ Variables } & \multicolumn{2}{|c|}{ Total } & \multicolumn{2}{|c|}{$\begin{array}{c}\text { Sin riesgo de } \\
\text { DMT" }\end{array}$} & \multicolumn{2}{|c|}{$\begin{array}{l}\text { Con riesgo de } \\
\text { DMT2 }\end{array}$} & \multicolumn{2}{|c|}{ Con DMT2 } \\
\hline & & \multirow[t]{2}{*}{$f$} & \multirow[t]{2}{*}{$\%$} & $f$ & $\%$ & $f$ & $\%$ & $f$ & $\%$ \\
\hline & & & & 147 & 60 & 66 & 26.9 & 32 & 13.1 \\
\hline \multirow[t]{4}{*}{ Interpersonal } & Apoyo Social & & & & & & & & \\
\hline & Alto & 176 & 71.8 & 104 & 70.7 & 51 & 77.3 & 21 & 65.6 \\
\hline & Bajo & 69 & 28.2 & 43 & 29.3 & 15 & 22.7 & 11 & 34.4 \\
\hline & Total & 245 & 100 & 147 & 100 & 66 & 100 & 32 & 100 \\
\hline \multirow[t]{8}{*}{ Institucional } & Ingreso Mensual & & & & & & & & \\
\hline & Suficiente & 161 & 65.7 & 106 & 72.1 & 38 & 57.6 & 17 & 53.1 \\
\hline & No Suficiente & 84 & 34.3 & 41 & 27.9 & 28 & 42.4 & 15 & 46.9 \\
\hline & Total & 245 & 100 & 147 & 100 & 66 & 100 & 32 & 100 \\
\hline & Estrés laboral & & & & & & & & \\
\hline & Sin estrés laboral & 235 & 95.9 & 140 & 95.2 & 66 & 100 & 29 & 90.6 \\
\hline & Con estrés laboral & 10 & 4.1 & 7 & 4.8 & 0 & 0 & 3 & 9.4 \\
\hline & Total & 245 & 100 & 147 & 100 & 66 & 100 & 32 & 100 \\
\hline \multirow[t]{4}{*}{ Comunitario } & Características comunitarias & & & & & & & & \\
\hline & Favorecedoras & 167 & 68.2 & 100 & 68.1 & 45 & 68.2 & 22 & 68.8 \\
\hline & No favorecedoras & 78 & 31.8 & 47 & 31.9 & 21 & 31.8 & 10 & 31.2 \\
\hline & Total & 245 & 100 & 147 & 100 & 66 & 100 & 32 & 100 \\
\hline \multirow[t]{8}{*}{ Política pública } & $\begin{array}{l}\text { Asistencia al servicio de salud } \\
\text { Cuando está enfermo }\end{array}$ & & & & & & & & \\
\hline & Cuando está sano & 212 & 86.5 & 128 & 87.1 & 57 & 86.4 & 27 & 84.4 \\
\hline & Total & 33 & 13.5 & 19 & 12.9 & 9 & 13.6 & 5 & 15.6 \\
\hline & & 245 & 100 & 147 & 100 & 66 & 100 & 32 & 100 \\
\hline & $\begin{array}{l}\text { Recomendación para hacer } \\
\text { ejercicio }\end{array}$ & & & & & & & & \\
\hline & $\mathrm{Si}$ & 164 & 66.9 & 92 & 62.6 & 49 & 74.2 & 23 & 71.9 \\
\hline & Tho & 81 & 33.1 & 55 & 37.4 & 17 & 25.8 & 9 & 28.1 \\
\hline & & 245 & 100 & 147 & 100 & 66 & 100 & 32 & 100 \\
\hline & $\begin{array}{l}\text { Recomendación sobre alimenta- } \\
\text { cion saludable }\end{array}$ & & & & & & & & \\
\hline & Si & 158 & 64.5 & 86 & 58.5 & 50 & 75.8 & 22 & 68.8 \\
\hline & INO & 87 & 35.5 & 61 & 41.5 & 16 & 24.2 & 10 & 31.2 \\
\hline & Iotal & 245 & 100 & 147 & 100 & 66 & 100 & 32 & 100 \\
\hline & Información sobre riesgo de & & & & & & & & \\
\hline & $\mathrm{Si}$ & 127 & 51.8 & 59 & 40.1 & 42 & 63.6 & 26 & 81.3 \\
\hline & Total & 118 & 48.2 & 88 & 59.9 & 24 & 36.4 & 6 & 18.7 \\
\hline & & 245 & 100 & 147 & 100 & 66 & 100 & 32 & 100 \\
\hline
\end{tabular}

Fuente: Elaboración propia

$\boldsymbol{n}=245$ 
no saludables, sobresaliendo la dieta y el sedentarismo; la presencia de SP/OB que redundan en la alteración de la producción y secreción de la insulina; la oxidación de ácidos grasos circulantes, la consecuente acumulación de glucosa sanguínea y la inadecuada calidad en los procesos de transducción y resistencia a la insulina.

El análisis de grupos con/sin riesgo y con cifras indicativas de DM2 según cifras de HbA1, permitió perfilar a los integrantes de la muestra. Los diagnosticados con DM2 fueron los de más edad, con menor ingreso económico y menor información sobre riesgo de DM2. Es bien conocido que el aumento en la edad se asocia con el riesgo de $\mathrm{DM}_{2}{ }^{(2,8)}$, debido a los procesos de envejecimiento que repercuten negativamente en los mecanismos fisiológicos compensatorios para mantener la calidad y cantidad en la excreción de insulina, teniendo como agravante la presencia de otros factores de riesgo (SB/OP, desequilibrio alimentario, sedentarismo) que exacerban el funcionamiento inadecuado en los proceso metabólicos relacionados con el equilibrio glucémico.

Se encontró que el ingreso económico suficiente para suplir las necesidades básicas, se presentó con mayor porcentaje en el subgrupo sin riesgo de DM2. El riesgo de DM2 está asociado con el bajo ingreso económico ${ }^{(41)}$. Es consistente con los hallazgos en el grupo de estudio, lo que indica que la capacidad para suplir las necesidades individuales y del hogar es vital en la promoción y consecución de estilos de vida saludables para evitar el desarrollo del riesgo de DM2.

La recepción de información sobre alimentación saludable fue mayor en el subgrupo con riesgo y con DM2. Se ha demostrado la asociación de la falta de información sobre el riesgo de DM2 y una inadecuada alimentación saludable con el riesgo de desarrollar DM2 ${ }^{(14,41)}$. Sin embargo, es posible que la recepción de información sobre alimentación saludable y sobre el riesgo de DM2 no genere efecto sobre la percepción del riesgo de enfermar y de ésta manera, no influya en el aumento de la consciencia en salud, lo que impide cambios positivos y duraderos para favorecer los estilos de vida saludables.

Con relación al respaldo teórico, las perspectivas de naturaleza ecológica han venido ganando aceptación como alternativas y abordajes que incluyen factores no lineales, cambiantes, impredecibles, moderadores y mediadores; tienen en cuenta las influencias del contexto para explicar la complejidad sobre los comportamientos en salud, la producción y progresión hacia la enfermedad. Debido a esto, es importante y necesario que las investigaciones en salud se sustenten en teorías que permitan mejorar la comprensión de los fenómenos en el contexto real, aumentar la validez de los resultados y contribuir a visualizar el comportamiento futuro y potencial impacto sobre la salud de las poblaciones ${ }^{(13)}$

Estos resultados deben ser tomados con cautelaya que el muestreo fue no probabilístico debido a la imposibilidad de contar con un marco muestral para la selección aleatoria de los participantes; no se incluyeron otros indicadores biológicos relacionados con el riesgo de DM2, como la insulina, la glucemia, la curva de tolerancia oral a la glucosa y el perfil de lípidos; el diseño transversal impidió realizar un seguimiento en el tiempo para determinar la incidencia de DM2, la tasa de conversión de los participantes en riesgo de DM2 a DM2 y determinar relaciones causales; además, limita las posibilidades de generalización de los hallazgos a otras poblaciones.

\section{CONCLUSIÓN}

Se concluye que $13.1 \%$ de los adultos tuvieron cifras de $\mathrm{HbA1c}$ indicativas de DM2 y 26.9\% cifras de riesgo para DM2.

Se encontraron factores de riesgo para DM2 predominantes en la muestra como la presencia de antecedentes familiares de la enfermedad, obesidad y bajo desarrollo de estilos de vida saludables.

Existieron diferencias significativas en la edad, la percepción de riesgo de DM2, el ingreso económico, la información relativa a la alimentación saludable y la relacionada con la prevenciòn del riesgo de $\mathrm{DM} 2$, entre los sub-grupos sin riesgo, con riesgo y con DM2.

Es importante la utilización de la perspectiva ecológica que apertura la comprensión de los procesos y factores de diferentes niveles de organización social involucrados en el riesgo de desarrollar DM2.

\section{CONFLICTO DE INTERESES}

Los autores declaran no presentan ningún conflicto de interés.

\section{FINANCIAMIENTO}

Los autores declaran no haber recibido ningún tipo de financiamiento para el estudio.

\section{REFERENCIAS BIBLIOGRÁFICAS}

1. Federación Internacional de Diabetes (FID). Atlas de la Diabetes de la FID [Internet]. FID. 2017 [citado el 1 de Agosto de 2018]. Disponible en: http://www. diabetesatlas.org/ resources/2017-atlas.html

2. Asociación Americana de Diabetes. Clasificación y diagnóstico de Diabetes: estándares de cuidado médicos en diabetes 2019. Diabetes Care [Internet]. 2019 [citado el 30 Febrero de 2019]; 42 (1): S13-\$28. Disponible en: https:// care.diabetesjournals.org/content/42/ Supplement_1/S13. full-text.pdf

3. Instituto Nacional de Estadística, Geografía e Informática (INEGI). Estadísticas vitales [Internet]. INEGI. 2018 [citado el 12 de Diciembre de 2018]. Disponible en: http://www.inegi.org. $\mathrm{mx} / \mathrm{est} / \mathrm{contenidos/proyectos/registros/vitales/mortalidad/}$ 
tabulados/ConsultaMortalidad.asp

4. Organización Mundial de la Salud (OMS). Top 10 causes of death [Internet]. OMS. 2016 [citado el 21 de Marzo de 2019]. Disponible en: http:// www.who.int/gho/mortality_burden_ disease/causes_death/top_10/en/

5. Centro Nacional de Programas Preventivos y Control de Enfermedades (CENAPRECE). Programa de acción específico: prevención y control de la diabetes mellitus 2013-2018. [Internet]. 2018 [citado el 18 de Enero de 2019] Disponible en: http://www.cenaprece.salud.gob.mx/interior/ PAES2013_2018.html

6. Centro Nacional de Programas Preventivos y Control de Enfermedades CENAPRECE. Estrategia estatal para la prevencióny el control del sobrepeso, la obesidad y la diabetes del estado de Nuevo León 2013-2018 [Internet]. 2018 [citado el 18 de Enero de 2019] Disponible en: www.cenaprece.salud. gob.mx/programas/.../EstrategiaSODOaxaca.pdf

7. Rojas-Martínez R, Basto-Abreu A, Aguilar-Salinas C A, Zárate-Rojas E, Villalpando S, Barrientos-Gutiérrez T. Prevalencia de diabetes por diagnóstico médico previo en México. Salud Pública de Méx [Internet]. Mayo de 2018 [citado el 28 de Enero de 2019]; 60(3): 224-232. Disponible en: http://dx.doi.org/10.21149/8566

8. DeFronzo RA. From the Triumvirate to the Ominous Octet: A New Paradigm for the Treatment of Type-2 Diabetes Mellitus [Internet]. Abril de 2009 [citado el 27 de Febrero de 2019]; 58 (4): 773-95. Disponible en: doi: 10.2337/db09-9028 9. Balk EM, Earley A, Raman G, Avendano EA, Pittas AG, Remington PL. Combined diet and physical activity promotion programs to prevent type 2 diabetes among persons at increased risk: a systematic review for the Community Preventive Services Task Force. Ann Intern Med [Internet]. Septiembre de 2015 [citado el 27 de Febrero de 2019]; 163 (6): 437-451. Disponible en: DOI: 10.7326/M15-0452

10. Stevens, JW, Khunti K, Harvey R, Johnson M, Preston L, Woods HB, Goyder E. Preventing the progression to type 2 diabetes mellitus in adults at high risk: a systematic review and network meta-analysis of lifestyle, pharmacological and surgical interventions. Diabetes Res Clin Pract [Internet]. Marzo de 2015 [citado el 27 de Febrero de 2019]; 107(3): 320-331. Disponible en: doi: 10.1016/j.diabres.2015.01.027

11. Burke NJ, Joseph G, Pasick RJ, Barker JC. Theorizing social context: rethinking behavioral theory. Health Educ Behav [Internet]. Octubre de 2009 [citado el 27 de Febrero de 2019]; 36(5): 55s-70s. Disponible en: https://www.ncbi. nlm. nih.gov/pubmed/19805791

12. Krieger $\mathrm{N}$. Theories for social epidemiology in the 21st century: an ecosocial perspective. Int J Epidemiol [Internet]. Agosto de 2001 [citado el 27 de Febrero de 2019]; 30 (4):
668-77. Disponible en: https://doi.org/10.1093/ije/30.4.668

13. McLeroy KR, Bibeau D, Steckler A, Glanz K. An ecological perspective on health promotion programs. Health Educ Q [Internet]. Diciembre de 1988 [citado el 17 de Enero de 2019]; 15(4):351-77. Disponibleen: DOI: 10.1177/109019818801500401

14. Instituto Nacional de Salud Pública. Encuesta Nacional de Salud y Nutrición 2012 [Internet]. 2012 [citado el 18 de Enero de 2019]. Disponible en: https://ensanut.insp.mx/

15. Kahl KG, Schweiger U, Correll C, Müller C, Busch ML, Bauer $\mathrm{M}$, et al. Depression, anxiety disorders, and metabolic syndrome in a population at risk for type 2 diabetes mellitus. Brain Behav [Internet]. Marzo de 2015 [citado el 1 de Marzo de 2019]; 5(3). Disponible en: doi: 10.1002/brb3.306.

16. Pan A, Wang Y, Talaei M, Hu FB, Wu T. Relation of active, passive, and quitting smoking with incident type-2 diabetes: a systematic review and meta-analysis. Lancet Diabetes Endocrinol [Internet]. Diciembre de 2015 [citado el 21 de Marzo de 2019]; 3 (12): 958-67. Disponible en: doi: 10.1016/ S2213-8587(15)00316-2

17. Kowall, B, Rathmann W, Stang A, Bongaerts B, Kuss O, Herder C, Meisinger C. Perceived risk of diabetes seriously underestimates actual diabetes risk: The KORA FF4 study. PLoS One [Internet]. Enero de 2017 [citado el 21 de Abril de 2019]; 12 (1): e0171152. Disponible en: doi: 10.1016/j. rpped.2014.11.003

18. Altevers J, Lukaschek K, Baumert J, Kruse J, Meisinger C, Emeny RT, et al. Poor structural social support is associated with an increased risk of Type-2 diabetes mellitus: findings from the MONICA/KORA Augsburg cohort study. Diabet Med [Internet]. Enero de 2016 [citado el 27 de Diciembre de 2019]; 33 (1): 47-54. Disponible en: doi: 10.1111/dme.12951.

19. Kivimaki M, Virtanen M, Kawachi I, Nyberg ST, Alfredsson L, Batty GD, Jokela M. Long working hours, socioeconomic status, and the risk of incident type 2 diabetes: a metaanalysis of published and unpublished data from 222120 individuals. Lancet Diabetes Endocrinol [Internet]. Enero de 2015 [citado el 27 de Diciembre de 2019]; 3 (1): 27-34. Disponible en: doi: 10.1016/\$2213-8587(14)70178-0

20. Nyberg ST, Fransson El, Heikkila K, Ahola K, Alfredsson L, Bjorner JB, et al. Job strain as a risk factor for type-2 diabetes: a pooled analysis of 124,808 men and women. Diabetes Care [Internet]. Agosto de 2014 [citado el 27 de Diciembre de 2019]; 37(8): 2268-75. Disponible en: https://www.ncbi. nlm. nih.gov/pubmed/25061139

21. Carnethon MR, Sanchez BN, Moore K, et al. Longitudinal Associations between Neighborhood Physical and Social Environments and Incident Type-2 Diabetes Mellitus: The 
Multi-Ethnic Study of Atherosclerosis (MESA). JAMA Intern Med [Internet]. Agosto de 2015 [citado el 27 de Marzo de 2019]; 175(8): 1311-20. Disponible en: doi:10.1001/ jamainternmed.2015.2691

22. Dalton AM, Jones AP, Sharp SJ, Cooper AJ, Griffin S, Wareham NJ. Residential neighborhood greenspace is associated with reduced risk of incident diabetes in older people: a prospective cohort study. BMC Public Health [Internet]. Noviembre de 2016 [citado el 27 de Marzo de 2019]; 16(1): 1171. Disponible en: doi: 10.1186/s12889-0163833-z

23. Hill JO, Galloway JM, Goley A, Marrero DG, Minners R, Montgomery B, et al. Scientific statement: Socioecological determinants of prediabetes and type-2 diabetes. Diabetes Care [Internet]. Julio de 2013 [citado el 27 de Marzo de 2019]; 36(8): 2430-9. Disponible en: https://doi.org/10.2337/ dc13-1161

24. Muller G, Harhoff R, Rahe C, Berger K. Inner-city green space and its association with body mass index and prevalent type 2 diabetes: a cross-sectional study in an urban German city. BMJ Open [Internet]. Enero de 2018 [citado el 27 de Marzo de 2019]; 8 (1): e019062. Disponible en: doi: 10.1136/ bmjopen-2017-019062

25. Walker RJ, Smalls BL, Campbell JA, Strom Williams JL, Egede LE. Impact of social determinants of health on outcomes for type-2 diabetes: a systematic review. Endocrine [Internet]. Septiembre de 2014 [citado el 10 de Marzo de 2019]; 47(1): 29-48. Disponible en: doi: 10.1007/s12020-0140195-0.

26. Muller G, Hartwig S, Greiser KH, Moebus S, Pundt N, Schipf $S$, et al. Gender differences in the association of individual social class and neighbourhood unemployment rate with prevalent type-2 diabetes mellitus: a cross-sectional study from the DIAB-CORE consortium. BMJ Open [Internet]. Junio de 2014 [citado el 10 de Marzo de 2019]; 3(6). Disponible en: doi: 10.1136/bmjopen-2013-002601.

27. Fajardo-Dolci G, Gutiérrez JP, García-Saisó S. Acceso efectivo a los servicios de salud: operacionalizando la cobertura universal en salud. Salud Pública Méx [Internet]. Marzo de 2015 [citado el 12 de Marzo de 2019]; 57(2): 180-186. Disponible en: http://www.scielo.org.mx/scielo. php?script=sci_arttext\&pid=S0036-36342015000200014

28. Hernández-González MM, Pérez Jl, Jiménez-Garcés $C$, Alvarado-Bravo BG, Vieyra-Reyes P. Conductas y factores que influyen en la utilización de servicios de salud en la población adulta de la región sur del Estado de México, México. Revista de Medicina e Investigación [Internet]. Julio de 2015 [citado el 12 de Marzo de 2019]; 1 (2): 86-94. Disponible en: https://www.elsevier.es/es-revista-revista- medicina-e-investigacion-353-articulo-conductas-factoresque-influyen-utilizacion-X2214310613085565

29. Clark CR, Ommerborn MJ, Hickson DA, Grooms KN, Sims M, Taylor HA, et al. Neighborhood disadvantage, neighborhood safety and cardiometabolic risk factors in African Americans: biosocial associations in the Jackson Heart study. PLoS One [Internet]. Mayo de 2013 [citado el 14 de Marzo de 2019]; 8 (5): e63254. Disponible en: doi: 10.1371/journal.pone.0063254

30. Tareque Ml, Koshio A, Tiedt AD, Hasegawa T. Are the rates of hypertension and diabetes higher in people from lower socioeconomic status in Bangladesh? Results from a nationally representative survey. PLoS One [Internet]. Mayo de 2015 [citado el 14 de Marzo de 2019]; 10 (5): e0127954. Disponible en: https://www.ncbi.nlm.nih.gov/ pubmed/26017066

31. Ley General de Salud. DOF. 2014 [actualizado el 04 de Junio de 2014; consultado 18 de Enero de 2019]. Disponible: https://mexico.justia.com/federales/leyes/ley-general-desalud/

32. Walker SN, Sechrist KR, Pender NJ. The healthpromoting lifestyle profile: development and psychometric characteristics. Nurs Res [Internet]. Marzo de 1987 [citado el 16 de Marzo de 2019]; 36 (2): 76-81. Disponible en: https:// www.ncbi.n/m.nih.gov/pubmed/3644262

33. Sousa, VD, Ryan-Wenger NA, Driessnack $M$, Jaber AF. Factorial structure of the perception of risk factors for type 2 diabetes scale: exploratory and confirmatory factor analyses. J Eval Clin Pract [Internet]. Diciembre de 2010 [citado el 16 de Marzo de 2019]; 16 (6): 1096-1102. Disponible en: doi: 10.1111/j.1365-2753.2009.01276.x.

34. Spielberger, CD, Gorsuch RL, Lushene R, Vagg PR, Jacobs GA. Manual for the state-trait anxiety inventory. Palo Alto, CA: Consulting Psychologists Press. 1983.

35. Beck, AT, Steer RA, Brown OK. Beck depression inventory manual (2nd ed.). San Antonio. TX: Psychological Corporation. 1996.

36. Zimet $G$, Dahlem $N$, Sara Z, Farley G. The multidimensional scale of perceived social support. J Pers Assess [Internet]. Enero de 1988 [citado el 16 de Marzo de 2019]; 52 (1): 30-41. Disponible en: https://psycnet.apa.org/ record/1988-18939-001

37. Siegrist J, Starke D, Chandola T, Godin I, Marmot M, Niedhammer I, Peter R. The measurement of effort-reward imbalance at work: European comparisons. Soc Sci Med [Internet]. Abril de 2004 [citado el 16 de Marzo de 2019]; 58 (8): 1483-1499. Disponible en: doi: 10.1016/S0277- 
38. Echeverría SE, Diez-Roux AV, Link BG. Reliability of selfreported neighborhood characteristics. J Urban Health [Internet]. Diciembre de 2004 [citado el 16 de Marzo de 2019]; 81 (4): 682-701. Disponible en: doi: 10.1093/jurban/ jth151

39. Programa de acción específico: prevención y control de la diabetes mellitus 2013-2018 Nuevo León. Centro Nacional de Programas Preventivos y Control de Enfermedades. CENAPRECE. 2013 [actualizado Diciembre 2015; citado el 18 de Enero de 2019]. Disponible en: https://www.gob. $\mathrm{mx} /$ salud/documentos/programa-de-accion-especificoprevencion-y-control-de-la-diabetes-mellitus-2013-2018

40. Castro-Juárez CJ, Ramírez-García SA, Villa-Ruano N, García-Cruz D. Epidemiología genética sobre las teorías causales y la patogénesis de la diabetes mellitus tipo 2. Gac Med Mex [Internet]. Mayo de 2017 [citado el 6 de Junio de 2019]; 153: 864-874. Disponible en: https:// www.researchgate.net/profile/Sergio_Ramirez_Garcia/ publication/318429726_Epidemiologia_genetica_sobre_ las_teorias_causales_y_la_patogenesis_de_la_diabetes_ mellitus_tipo_2/links/5aa93b70aca272d39cd50833/ Epidemiologia-genetica-sobre-las-teorias-causales-y-lapatogenesis-de-la-diabetes-mellitus-tipo-2.pdf

41. Rivera LA, Lebenbaum M, Rosella LC. The influence of socioeconomic status on future risk for developing Type 2 diabetes in the Canadian population between 2011 and 2022: differential associations by sex. Int J Equity Health [Internet]. Octubre de 2015 [citado el 6 de Junio de 2019]; 14: 101. Disponible en: doi: 10.1186/s12939-015-0245-0 